\title{
Patrones espaciales en un herbazal halófilo: suelos, comunidades vegetales y plasticidad fenotípica de Sporobolus virginicus (L.) Kunth, Révis. Gramin. 1: 67 (1829) en el Estado Miranda, Venezuela
}

Spatial patterns in a halophyte grassland: soils, plant communities and phenotypic plasticity of Sporobolus virginicus (L.) Kunth, Révis. Gramin. 1: 67 (1829) in Miranda state, Venezuela

\section{Yamilex Avendaño, Irene C. Fedón, Eduardo M. Barreto-Pittol, María de los Ángeles Marrero, María B. Barreto y Lourdes M. Suárez-Villasmil}

\section{Resumen}

Se estudió la asociación entre los cambios del sustrato y la vegetación en un herbazal halófilo dominado por Sporobolus virginicus. Se cuantificaron la densidad aparente $\left(\mathrm{g} / \mathrm{cm}^{3}\right)$, la materia orgánica $(\%)$, el carbono inorgánico (\%), el pH, la textura, la salinidad del suelo (\%) y la salinidad intersticial (\%o), así como la cobertura relativa de cada especie (\%), la cobertura total $(\%)$ y la biomasa total $\left(\mathrm{g} / \mathrm{m}^{2}\right)$ de la vegetación, en 42 unidades de muestreo ubicadas en un transecto de 66 metros de longitud. La comunidad conformada por 7 especies, estuvo dominada por 2 morfotipos de S. virginicus. El morfotipo grande (probablemente variedad virginicus) estuvo asociado con suelos de mayor $\mathrm{pH}$, mayor densidad aparente, menor salinidad y menor porcentaje de materia orgánica, en comparación con el morfotipo pequeño (probablemente variedad minor). $\mathrm{El} \mathrm{pH}$, el porcentaje de materia orgánica, la salinidad y la densidad aparente determinaron significativamente $(p<0,001) 26 \%$ de la variación explicada en la comunidad. Sobre el transecto se conformaron 3 zonas con características edáficas distintas, en las cuales se establecieron distintas comunidades herbáceas con algunas especies compartidas. La dinámica del agua en el suelo podría responder a pequeñas variaciones en la microtopografía, lo que determinaría diferencias en los procesos de inundación y evaporación a escala local.

Palabras clave. Estado Miranda. Herbáceas halófilas. Humedales. Suelos hídricos.

\begin{abstract}
The relationship between soil and vegetation was studied in a salt marsh dominated by Sporobolus virginicus (L.) Kunth (Poaceae). We used 42 sampling units placed on a $66 \mathrm{~m}$ long transect; in each soil sample we measured the bulk density $\left(\mathrm{g} / \mathrm{cm}^{3}\right)$, organic matter $(\%)$, inorganic carbon $(\%), \mathrm{pH}$, texture, soil salinity (\%o) and interstitial salinity (\%o). Also, the relative cover of each species (\%), total vegetation cover $(\%)$, total biomass of the vegetation $\left(\mathrm{g} / \mathrm{m}^{2}\right)$, and the relative composition $(\%)$ of each species was measured. The community consisted of 7 species and was dominated by 2 S. virginicus morphotypes: a large morphotype (probably virginicus variety) was associated with soils characterized by higher
\end{abstract}


$\mathrm{pH}$, higher bulk density, lower salinity and lower organic matter (\%), in comparison with the small morphotype (probably minor L. M. Bailey variety). The $\mathrm{pH}$, organic matter, salinity and bulk density determined significantly $(\mathrm{p}<0.001$ ) $26 \%$ of explained variance of this community. The transect showed three zones with different edaphic characteristics and different herbaceous communities; nevertheless, those communities had some common species. The water dynamic of the soil may respond to small topographic variations, which could in turn determine differences on local flood and evaporation processes.

Keywords. Herbaceous halophytes. Hydric soils. Miranda state. Wetlands.

\section{Introducción}

Los herbazales halófilos están asociados con ambientes costeros en los cuales hay acumulación de sedimento, crecimiento de angiospermas (Pennings y Bertness, 2001) y un amplio espectro de gradientes ambientales que dan como resultado la zonación de las plantas (Lonard et al., 2011). Pueden presentar suelos inundados o saturados por agua de manera permanente o estacional (Mitsch y Gosselink, 2007; State of Florida, 2011: Everglades forever, Glossary of Terms, http:// www.dep.state.fl.us/evergladesforever/about/ glossary.htm\#w) y funcionan como ambientes de transición entre ecosistemas marinos $y$ terrestres. Son considerados biodiversos por sus especies, condiciones y procesos biogeoquímicos (Mitsch y Gosselink, 2007; Reddy y DeLaune, 2008), constituyen hábitats críticos para especies amenazadas y funcionan como centros de especiación (Mitsch y Gosselink, 2007; Figueroa et al., 2016). En los herbazales halófilos sometidos a inundación por agua dulce y/o la marea, la topografía y la hidrología son los principales factores con efecto sobre los cambios del sustrato (Reddy y DeLaune, 2008; Kim y Yu, 2009), ya que determinan variaciones en la humedad, el $\mathrm{pH}$ y estado de oxidación de las especies químicas (Pennings y Callaway, 1992; van de Rijt et al., 1996; Gordon et al., 2007; Mitsch y Gosselink, 2007; Reddy y DeLaune, 2008), la disponibilidad de nutrientes (Güsewell, 2005; van Diggelen et al., 2015), la descomposición de la materia orgánica (Torres et al., 2012) y la salinidad (Reddy y DeLaune, 2008; Lonard et al., 2011); de modo que la presencia, profundidad y duración de la lámina de agua produce cambios en la biogeoquímica y genera variaciones espaciotemporales en las condiciones del suelo, dependiendo de la exposición a una lámina de agua con características específicas (Reddy, 1993; Barker y Maltby, 2009). En los herbazales halófilos, los gradientes locales son más relevantes que los procesos regionales en la estructuración de sus comunidades, que son descritas como relativamente simples y conformadas por especies con amplia distribución geográfica (Guo et al., 2015).

Las adaptaciones de las especies que crecen en herbazales halófilos responden a las constantes fluctuaciones de los factores ambientales (Mitsch y Gosselink, 2007), especialmente la salinidad (Sharpe y Baldwin, 2009; Lonard et al., 2011). Una de las respuestas adaptativas de las plantas ante la variabilidad ambiental de estos ecosistemas es la plasticidad fenotípica, que permite la tolerancia a variaciones ambientales, incluidas aquellas asociadas con cambios climáticos (Gratani, 2014). Cabrera et al. (2013) identificaron en la zona de estudio la presencia de dos morfotipos de Sporobolus virginicus (L.) Kunth, los que a partir de su morfología, es posible que correspondan con alguna de las variedades de esta especie reportadas para Australia y Estados Unidos (Smith-White, 1979, 1988). El morfotipo pequeño (probablemente S. virginicus var. minor L.M. Bailey) presenta menos inflorescencias, más tallos y más estolones, así como culmos más cortos de aproximadamente la mitad del diámetro, en comparación con el morfotipo grande (probablemente S. virginicus var. virginicus). Teniendo en cuenta las condiciones de estos ambientes, se hace la predicción de que si 
hay cambios en el sustrato, estos deben determinar la distribución de las comunidades herbáceas y posiblemente estar relacionados con la plasticidad fenotípica de Sporobolus virginicus. De esta forma, este trabajo desarrolló una caracterización de los suelos, así como de la estructura y la composición florísticas de la vegetación, en un herbazal halófilo adyacente a un manglar que tiene influencia de una laguna de agua dulce de poca profundidad, y está cubierto en gran parte por un herbazal de plantas emergentes y flotantes.

\section{Materiales y métodos}

Área de estudio. El herbazal halófilo se ubica en la laguna La Reina, municipio Brión, estado Miranda, Venezuela $\left(10^{\circ} 31^{\prime} 20^{\prime \prime} \mathrm{N}-66^{\circ} 07^{\prime} 53^{\prime \prime} \mathrm{O}\right.$; 3 m s.n.m.); la laguna está localizada entre los poblados de Higuerote, Carenero y Curiepe (Figura 1).

El uso predominante del área es turísticorecreacional, urbano y agrícola; y los usos asociados son protector, portuario, seguridad y defensa, así como obras de infraestructuras (almacenaje de petróleo, aeropuerto de Higuerote y canales de navegación) (CEOTEBM, 2010). La precipitación promedio anual es de $1135 \mathrm{~mm}$, producto de los vientos alisios cargados de humedad que penetran por la zona costera, con variaciones entre los 1000 y 1500 mm/año. El clima es biestacional, con un periodo de lluvia entre agosto y noviembrediciembre. Las temperaturas máxima y mínima son $27{ }^{\circ} \mathrm{C}$ y $18{ }^{\circ} \mathrm{C}$ respectivamente, con un promedio anual de $25^{\circ} \mathrm{C}$ (Barreto et al., 2001).

La red hidrográfica que aporta agua dulce a este humedal posee un patrón de drenaje dependiente de la geología: el río Curiepe (régimen permanente) y la quebrada El Oso (régimen desconocido) aportan agua dulce a la laguna Loma del Viento, y esta a su vez a la laguna La Reina; por otro lado, la quebrada Los Flores (régimen desconocido) aporta agua dulce directamente a la laguna La Reina (CEOTEBM, 2010).

El relieve es plano, con una pendiente menor a $10^{\circ}$ y alturas que no superan los $40 \mathrm{~m}$, siendo una planicie aluvial reciente y un extenso plano costero (CEOTEBM, 2010). La vegetación está conformada por manglares litorales y estuarinos, así como por vegetación halófila que cubre las áreas intermareales y las lagunas costeras. En la actualidad, los bosques que rodean la laguna han estado bajo una intensa presión humana debido al desarrollo turístico y recreacional de la región (Barreto et al., 2001) con presencia de actividades urbanas, recreativas, agrícolas y ganaderas (Suárez-Villasmil et al., 2015).

El herbazal halófilo se localiza entre un humedal de macrófitas emergentes, dominado por Cyperus articulatus L. (Cyperaceae) y un bosque de manglar dominado por Avicennia germinans L. (Acanthaceae) con presencia de Rhabdadenia biflora (Jacq.) Müll. Arg. (Apocynaceae) y planicies salinas desprovistas de vegetación (Figura 2).

Trabajo de campo. El muestreo fue realizado en agosto de 2013 en un herbazal halófilo dominado por Sporobolus virginicus. Se definió un transecto de $66 \mathrm{~m}$ de longitud, con 14 puntos separados entre sí por una distancia de $4 \mathrm{~m}$. Este transecto se orientó en dirección norte (punto 1) a sur (punto 14), aproximadamente (Figura 1). En cada punto del transecto se establecieron tres parcelas de 1 $\mathrm{m}^{2}$, separadas $2 \mathrm{~m}$ entre sí, por lo que a nivel de cada punto se estudiaron tres franjas: la franja A, con ubicación más cercana a una planicie salina, un manglar dominado por Avicennia germinans y un arbustal (Figura 1), la franja B, intermedia y topográficamente más elevada, y la franja $C$, más cercana a un humedal dominado por Cyperus articulatus. En total, se colocaron 42 unidades de muestreo.

Todas las parcelas de $1 \mathrm{~m}^{2}$ fueron georreferenciadas con un receptor de posicionamiento global (GPS) Garmin ${ }^{\circledR}$, modelo eTrex Vista Cx, con precisión de $\pm 5 \mathrm{~m}$. En cada parcela se colectó una muestra de suelo en los primeros $20 \mathrm{~cm}$ de profundidad para la cuantificación de las variables fisicoquímicas, y otra muestra para determinación de la densidad aparente. Se tomaron cuatro 


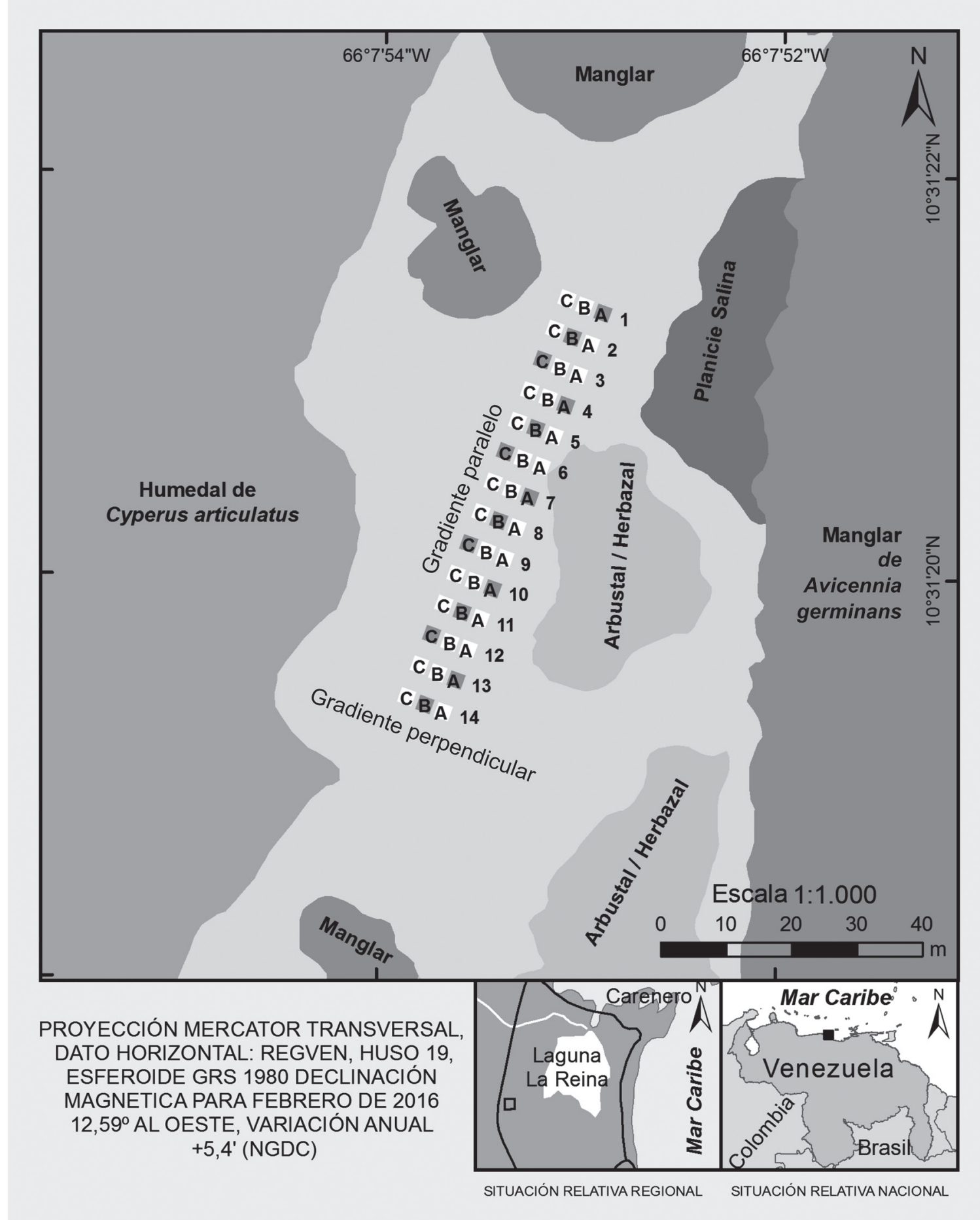

Figura 1. Área de estudio: laguna La Reina, municipio Brión, estado Miranda. Se presentan las unidades de vegetación, las unidades de muestreo (recuadros) así como la ubicación relativa de los dos gradientes: paralelo al manglar de Avicennia germinans y al herbazal de Cyperus articulatus se encuentra un gradiente orientado entre los puntos 1 y 14 . Perpendicular a este, se establecieron las franjas: A) más cercana al manglar, B) intermedia y C) más cercana al humedal de Cyperus articulatus. La biomasa fue cosechada sistemáticamente solo en las unidades sombreadas. 

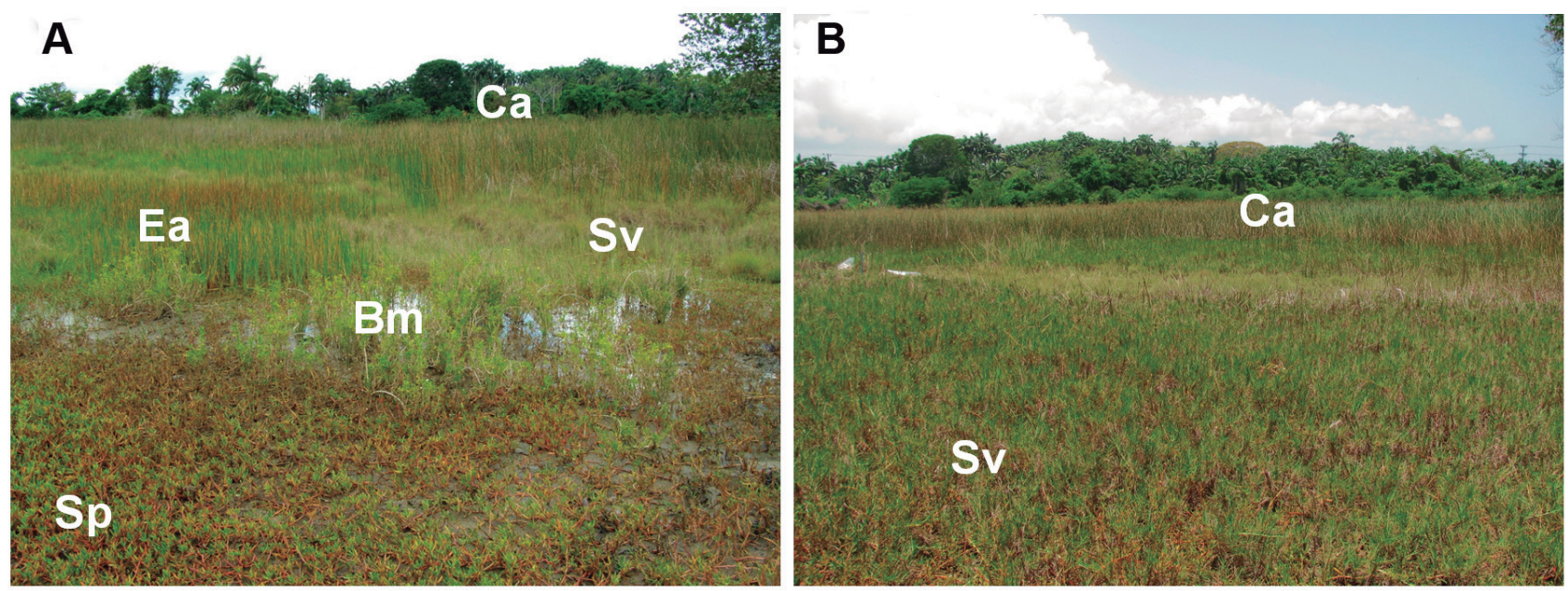

Figura 2. Fotografías del herbazal estudiado donde se observan las franjas o zonas de vegetación. A) El humedal presenta lámina de agua, en primer plano se observa Sesuvium portulacastrum ( $\mathrm{Sp}$ ) seguido de Batis marítima $(\mathrm{Bm})$ y luego a la izquierda un parche de Eleocharis acutangula (Ea) y a la derecha de Sporobolus virginicus (Sv, ambos morfotipos). Al fondo un humedal leñoso o arbóreo con palmas del género Roystonea detrás de una franja de Cyperus articulatus (Ca). B) Al frente de esta visual se observa un parche de Sporobolus virginicus (Sv, ambos morfotipos) y luego un parche de Cyperus articulatus $(\mathrm{Ca})$.

muestras de agua intersticial por punto de muestreo (a una profundidad de $10 \mathrm{~cm}$ ), a una distancia de $50 \mathrm{~cm}$ entre sí y a $25 \mathrm{~cm}$ del borde de la parcela. Las muestras de suelo fueron colocadas en bolsas plásticas, y las muestras de agua en envases plásticos, para su preservación, transporte y posterior tratamiento en el laboratorio.

Tratamiento de las muestras. Las muestras de suelo fueron colocadas en envases de plástico y secadas en una estufa a $50{ }^{\circ} \mathrm{C}$. Posteriormente fueron fragmentadas, tamizadas (usando una apertura de malla de $2 \mathrm{~mm}$ ) y homogeneizadas para los análisis de laboratorio. La determinación de la textura, se realizó aplicando el método de Bouyoucos (1962), con una solución de pirofosfato de sodio $(0,02 \mathrm{~N})$ como dispersante.

Para determinar la densidad aparente, se tomó una muestra de los primeros $10 \mathrm{~cm}$ del suelo con tubos de policloruro de vinilo (PVC) de 5,1 cm de diámetro y $10 \mathrm{~cm}$ de longitud. Se determinó el peso húmedo de la muestra contenida en el cilindro así como el peso seco luego de colocarlo en una estufa con ventilación forzada a $50{ }^{\circ} \mathrm{C}$, hasta llevarlo a peso constante. La densidad aparente $\left(\mathrm{g} / \mathrm{cm}^{3}\right)$ se calculó como la relación entre el peso seco de la muestra y el volumen del cilindro que la contenía.

La salinidad intersticial fue cuantificada con un refractómetro digital $\mathrm{ATAGO}^{\circledR}$ precisión $\pm 1 \%$, en muestras extraídas a $10 \mathrm{~cm}$ de profundidad. El pH y la salinidad de las muestras de suelo fueron determinados hidratando $3 \mathrm{~g}$ de suelo seco, con $15 \mathrm{~mL}$ de agua desionizada, en una relación 1:5, agitando la mezcla manualmente por cinco minutos y dejando reposar por un periodo mayor a 14 horas. En el líquido sobrenadante se realizaron las medidas de salinidad con un instrumento $\mathrm{ATAGO}^{\circledR}$ de precisión 0,1% y pH con un instrumento HANNA ${ }^{\circledR}$ (modelo 991301), de precisión $\pm 0,01$; y para la salinidad $\pm 2 \%$ F.S (Full scale).

Utilizando calentamiento progresivo en una mufla, se realizaron determinaciones de materia orgánica y carbonato inorgánico $\left(\mathrm{CO}_{3}{ }^{2-}\right)$ utilizando el método de pérdida por ignición (Loss on Ignition, LOI). Para ello fueron utilizados crisoles de porcelana donde se pesó aproximadamente $1 \mathrm{~g}$ 
de muestra. El porcentaje de materia orgánica se determinó a $550^{\circ} \mathrm{C}$ por 2,5 horas y el porcentaje de carbonato inorgánico a $950{ }^{\circ} \mathrm{C}$ por 2,5 horas (Hieri et al., 2001).

Muestreo de vegetación. En cada parcela de $1 \mathrm{~m}^{2}$ se estimó in situ el porcentaje de cobertura aérea (biomasa total y por especie). Adicionalmente, se cosechó la biomasa en cada punto, utilizando una subunidad muestral de $0,25 \mathrm{~m}^{2}$ en las parcelas seleccionadas de $1 \mathrm{~m}^{2}$ (Figura 1). El material vegetal fue almacenando en una bolsa de papel y pesado in situ, posterior a lo cual se separó manualmente la biomasa verde y seca. Las muestras se secaron en una estufa con ventilación forzada a $35^{\circ} \mathrm{C}$ hasta peso constante.

Adicionalmente fueron colectados especímenes botánicos para su posterior identificación; que se llevó a cabo empleando claves taxonómicas (Conard, 1905; Velázquez, 1994; Rial, 2009), así como a través de la comparación con material del Herbario Nacional de Venezuela (VEN) y del herbario del Laboratorio de Ecología de Plantas Acuáticas (LEPA), Instituto de Zoología y Ecología Tropical (IZET), Facultad de Ciencias, Universidad Central de Venezuela (UCV). Los morfotipos de Sporobolus virginicus fueron identificados de acuerdo con las muestras testigo empleadas por Cabrera et al. (2013), las cuales están referidas en el Herbario Nacional de Venezuela (VEN) con las siguientes identificaciones: "Steyermark y Manara 110330" (morfotipo grande) y “Aristeguieta 448" (morfotipo pequeño).

Procesamiento estadístico. Las asociaciones entre las variables del sustrato se midieron con el coeficiente de correlación r de Spearman (Daniel, 2002). Las diferencias en los parámetros del sustrato fueron determinadas con la prueba de Kruskal-Wallis (Zar, 1999); y en los casos donde hubo al menos un valor distinto $(\mathrm{p}<0,05)$, fue aplicada la prueba a posteriori de Mann-Whitney con la corrección de Bonferroni (Hammer et al., 2001). Las probabilidades derivadas de la prueba a posteriori son mencionadas cuando hay valores significativamente distintos.
La composición de especies fue estudiada a través del promedio del porcentaje de cobertura relativa de cada especie. El análisis de la composición florística sobre el transecto sugirió la diferenciación del área de muestreo en tres zonas. La confirmación de este resultado fue hecha con un Análisis discriminante (Afifi y Clark, 1997), sobre las variables estandarizadas del suelo que mostró algún cambio en dicho transecto. Posteriormente, se aplicó un Análisis de Similitudes o ANOSIM (Clarke, 1993) que produce un valor alto del estadístico $R(p<0,05)$ en presencia de comunidades distintas. El ANOSIM midió las similitudes entre comunidades con la distancia Bray-Curtis (Anderson, 2001). La prueba a posteriori asociada con el ANOSIM permitió identificar comunidades distintas entre sí.

Para medir la asociación de los morfotipos de Sporobolus virginicus se aplicó la prueba de Jicuadrado $\left(\chi^{2}\right)$ bajo la hipótesis de independencia (Daniel, 2002). Se reportó, además, el valor del coeficiente "Phi" (Yule, 1912) que estandariza el valor de Ji-cuadrado para que varíe entre cero y uno, mostrando la magnitud de la asociación.

Para evaluar la forma como los factores edáficos determinan la estructura comunitaria, se aplicó un Análisis de Correspondencia Canónica (CCA) (Ter Braak, 1986), cuyo porcentaje de varianza explicada es proporcional al nivel de determinación que tienen las variables ambientales sobre la estructura de la comunidad (Palmer, 1993). Todos los análisis y gráficos fueron realizados con el programa PAST (Hammer et al., 2001) versión 3.13 (Agosto 2016).

\section{Resultados}

Cobertura, biomasa, composición y riqueza de la vegetación. Entre franjas $(A, B, C)$ solo se detectaron cambios en la biomasa total (Tabla 1), ya que la franja A mostró menor biomasa total $(412,4$ $\left.\mathrm{g} / \mathrm{m}^{2}, \mathrm{p}<0,09\right)$ que las franjas B y C $\left(828,3 \mathrm{~g} / \mathrm{m}^{2}\right.$ promedio). Al estudiar los atributos estructurales sobre el transecto (por puntos), solo los puntos extremos difirieron $(p<0,05)$ en su cobertura 
total (punto 1: 51,6 \%; punto 14: 95,1\%), pero no hubo diferencias entre puntos consecutivos del transecto. El resto de los atributos estructurales no se comparó estadísticamente porque solo se cosechó una unidad de biomasa por punto.

Se encontraron en total siete especies herbáceas con un porcentaje de cobertura relativa muy desigual (Tabla 2). Sporobolus virginicus fue dominante $(85,74 \%)$ y estuvo representada por los dos morfotipos referidos, el grande (probablemente S.virginicus var. virginicus) ligeramente con mayor cobertura que el pequeño (probablemente $S$. virginicus var. minor). Eleocharis acutangula (Roxb.) Schult. (Cyperaceae) fue la segunda especie con mayor cobertura y el resto de las especies fueron muy escasas, con coberturas inferiores al $2 \%$. La distribución de las especies sobre las franjas (Tabla 2) muestra que Sporobolus virginicus (ambos morfotipos) y Eleocharis acutangula estuvieron presentes en las tres franjas, pero su cobertura relativa varió ligeramente de una franja a otra. Typha domingensis Pers. (Typhaceae) no apareció en la franja $\mathrm{C}$ y Cyperus articulatus no apareció en la franja A, la más lejana al humedal dominado por esta especie.

Tabla 1. Media, mínimo y máximo de los atributos estructurales de la vegetación así como los parámetros del sustrato. Se presentan los niveles de significancia $(\mathrm{p})$ para evaluar diferencias por parcelas $(\mathrm{A}, \mathrm{B}, \mathrm{C})$, por puntos (1 al 14) y por zonas $(1,2,3)$. La significancia para las variables marcadas con * no se pudo calcular porque solo se cosechó una muestra de biomasa por punto.

\begin{tabular}{|c|c|c|c|c|c|c|}
\hline Variables & $\mathbf{n}$ & Media & Min-Max & $\begin{array}{c}\mathrm{p} \\
(\text { Parcela } A, B, C)\end{array}$ & $\begin{array}{c}\mathrm{p} \\
\text { (Punto } 1 \text { al } \\
14)\end{array}$ & $\begin{array}{c}\mathrm{p} \\
\text { (Zonas, } 1,2, \\
3)\end{array}$ \\
\hline Cobertura total (\%) & 42 & 76,6 & $(41,6-100)$ & 0,64 & 0,01 & 0,00 \\
\hline Biomasa verde $\left(\mathrm{g} / \mathrm{m}^{2}\right)$ & 14 & 84,0 & $(15,5-222,2)$ & 0,06 & * & 0,91 \\
\hline Biomasa seca $\left(\mathrm{g} / \mathrm{m}^{2}\right)$ & 14 & 87,3 & $(39,4-188,6)$ & 0,08 & * & 0,59 \\
\hline Biomasa total $\left(\mathrm{g} / \mathrm{m}^{2}\right)$ & 14 & 171,3 & $(54,8-410,8)$ & 0,05 & * & 0,89 \\
\hline Relación verde/seco & 14 & 1,0 & $(0,4-2,5)$ & 0,42 & * & 0,29 \\
\hline Densidad aparente $\left(\mathrm{g} / \mathrm{cm}^{3}\right)$ & 42 & 1,2 & $(0,9-1,4)$ & 0,43 & 0,07 & 0,05 \\
\hline Materia orgánica (\%) & 42 & 2,7 & $(1,6-3,7)$ & 0,25 & 0,02 & 0,02 \\
\hline Carbono inorgánico (\%) & 42 & 1,7 & $(1,1-2,0)$ & 0,98 & 0,10 & 0,08 \\
\hline pH del suelo & 42 & 7,7 & $(7,3-8,1)$ & 0,00 & 0,03 & 0,00 \\
\hline Arena (\%) & 42 & 68,0 & $(59,3-78,0)$ & 0,05 & 0,33 & 0,13 \\
\hline Limo (\%) & 42 & 30,7 & $(20,0-39,6)$ & 0,04 & 0,39 & 0,14 \\
\hline Arcilla (\%) & 42 & 1,3 & $(0,0-4,4)$ & 0,61 & 0,67 & 0,68 \\
\hline Salinidad suelo (\%o) & 42 & 8,3 & $(5,0-12,5)$ & 0,60 & 0,11 & 0,004 \\
\hline Salinidad intersticial (\%o) & 42 & 21,7 & $(14,8-28,8)$ & 0,42 & 0,51 & 0,29 \\
\hline
\end{tabular}


Tabla 2. Composición florística y cobertura promedio (\%) de las especies presentes en el herbazal halófilo. Fueron delimitadas tres franjas: A) más cercanas al manglar, B) intermedias) y C) adyacentes al humedal dominado por Cyperus articulatus.

\begin{tabular}{|c|c|c|c|c|c|}
\hline Nombre científico & Familia & $\begin{array}{c}\text { Franja } \\
\text { A }\end{array}$ & $\begin{array}{c}\text { Franja } \\
\text { B }\end{array}$ & $\begin{array}{l}\text { Franja } \\
\text { C }\end{array}$ & Total \\
\hline Rhabdadenia biflora (Jacq) Mull.Arg & Apocynaceae & 0,02 & 0,00 & 0,00 & 0,01 \\
\hline Batis maritima $\mathrm{L}$. & Bataceae & 0,03 & 0,00 & 0,00 & 0,01 \\
\hline Sesuvium portulacastrum (L.) L. & Aizoaceae & 0,34 & 0,00 & 0,00 & 0,11 \\
\hline Typha domingensis Pers. & Typhaceae & 0,20 & 1,38 & 0,00 & 0,52 \\
\hline $\begin{array}{l}\text { Sporobolus virginicus L. Kunth - Morfotipo } \\
\text { grande }\end{array}$ & Poaceae & 47,48 & 37,05 & 47,95 & 44,16 \\
\hline $\begin{array}{l}\text { Sporobolus virginicus L. Kunth - Morfotipo } \\
\text { pequeño }\end{array}$ & Poaceae & 33,71 & 50,53 & 40,49 & 41,58 \\
\hline Eleocharis acutangula (Roxb.) Schult. & Cyperaceae & 17,72 & 8,94 & 9,83 & 12,16 \\
\hline Cyperus articulatus L. & Cyperaceae & 0,00 & 2,11 & 1,73 & 1,28 \\
\hline
\end{tabular}

La cobertura de las tres especies más abundantes evidenció una estructuración natural del transecto en tres regiones (Figura 3), diferenciándose la zona 1, entre los puntos 1 y 5; la zona 2, del 6 al 10 y la zona 3, del 11 al 14. El morfotipo pequeño fue dominante en las zonas 1 y 3 y estuvo prácticamente ausente en la zona 2, mientras que el morfotipo grande fue dominante en la zona 2, pero tuvo baja abundancia en la zona 1 y prácticamente estuvo ausente en la zona 3. La frecuencia de aparición de estos morfotipos mostró una dependencia negativa $\left(X^{2}[v=1\right.$, $\mathrm{n}=672]=284,694 ; \mathrm{p}<0,0001)$, que produjo un valor del coeficiente Phi $\Phi=-0,651(p<0,0001)$. Este valor negativo y de magnitud media-alta refleja, en primer lugar, que existe algún tipo de exclusión importante entre los dos morfotipos, y en segundo lugar, que aunque los morfotipos tienden a excluirse, pueden también aparecer en la misma unidad muestral. El análisis de similitudes (ANOSIM) produjo como resultado un $\mathrm{R}=0,427(\mathrm{p}<0,0001)$ que indica que al menos una de las zonas constituye una comunidad distinta de las demás. En este caso, la prueba a posteriori para el ANOSIM evidenció que las tres zonas constituyeron comunidades distintas $(\mathrm{p}<$ 0,0066).

Caracterización del suelo. La densidad aparente, el carbono inorgánico, el contenido de arcilla, la salinidad intersticial y la salinidad del suelo, no mostraron diferencias significativas (Tabla 1). El pH presentó variaciones sobre las franjas, siendo más básico en la franja $B(7,9 ; p<0,003)$ con respecto a las franjas A y C (promedio=7,3). Asimismo, el porcentaje de limo fue más bajo en las franjas B $(29 \%, p<0,03)$ en relación con los valores hallados en A y C (promedio = $32 \%$ ). El porcentaje de arena fue mayor en la franja B $(70 \%, \mathrm{p}<0,02)$ al comparar con los valores hallados en $\mathrm{A}$ y $\mathrm{C}$ (promedio $=67 \%$ ), lo que evidencia que a nivel de las franjas, la región intermedia (B) presentó $\mathrm{pH}$ ligeramente más básico y suelos ligeramente más arenosos que la las franjas A y C.

Al evaluar los parámetros a lo largo del transecto, se observaron diferencias en el $\mathrm{pH}$ del suelo y en el porcentaje de materia orgánica, pero la prueba a posteriori no detectó tratamientos diferentes. Debido a la inconsistencia de este resultado, se 


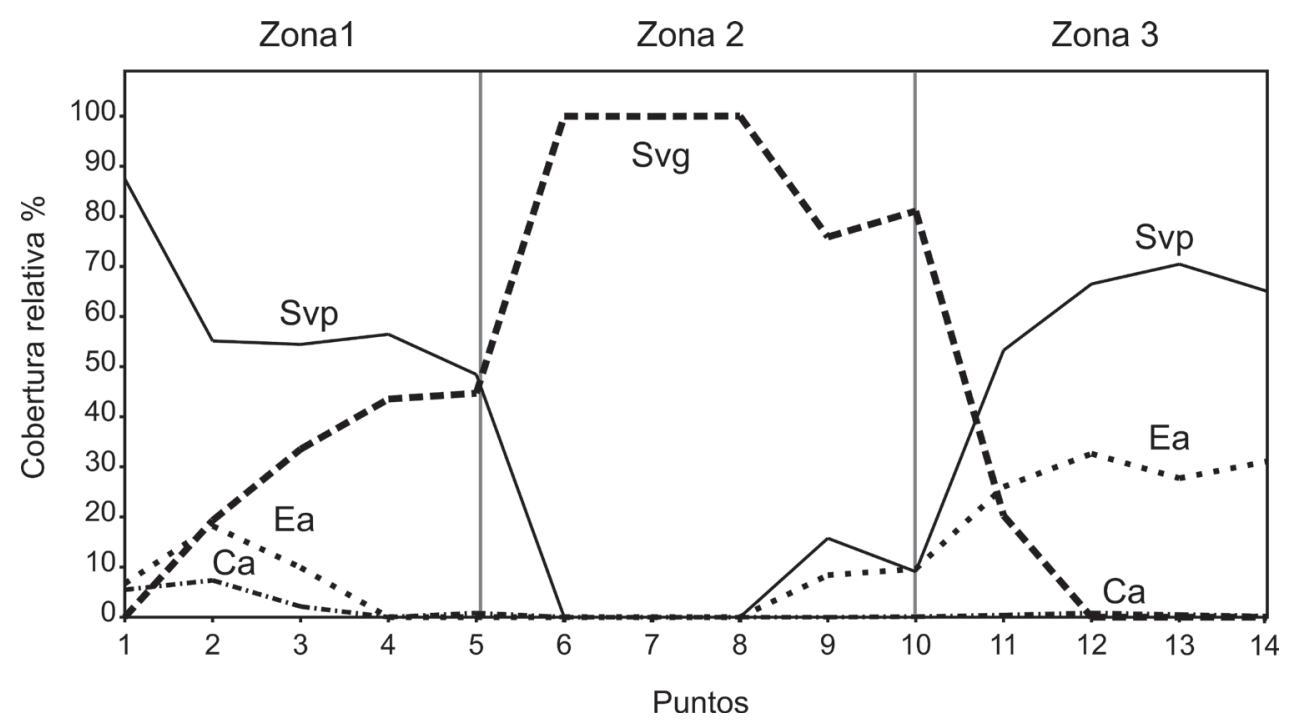

Figura 3. Cobertura de Sporobolus virginicus morfotipo pequeño (Svp), Sporobolus virginicus morfotipo grande (Svg), Eleocharis acutangula (Ea) y Cyperus articulatus (Ca) a lo largo de los 14 puntos del muestreo. La distribución de estas especies hizo evidente la presencia de tres zonas naturales.

decidió explorar las variaciones a nivel de las zonas (Figura 3), encontrando que la densidad aparente en la zona 2 fue mayor $\left(1,2 \mathrm{~g} / \mathrm{cm}^{3} ; \mathrm{p}<\right.$ 0,02 ) que en las zonas 1 y 3 (promedio $=1,0 \mathrm{~g} /$ $\mathrm{cm}^{3}$ ) (Tabla 1); el $\mathrm{pH}$ del suelo en la zona 2 fue mayor $(7,9 ; \mathrm{p}<0,02)$ en comparación con las zonas 1 y 3 (promedio $=7,6$ ); la salinidad de la zona 1 fue mayor $(9,6 \%$; p < 0,02) que la de las zonas 2 y 3 (promedio 7,8 \%o); y el porcentaje de materia orgánica de la zona 3 fue mayor $(2,5 \% ; p<0,01)$ que el de las zonas 1 y 2 (promedio $=1,8 \%$ ).

El análisis discriminante realizado con la densidad aparente, porcentaje de materia orgánica, $\mathrm{pH}$ y salinidad del suelo produjo $79 \%$ de clasificación correcta entre las tres zonas, lo que evidencia condiciones edáficas distintas con una diferenciación gradual entre ellas (Figura 4). La zona 1 estuvo caracterizada por mayor salinidad, menor densidad aparente, menor $\mathrm{pH}$ y niveles de materia orgánica que variaron de intermedios a altos. La zona 2 presentó un $\mathrm{pH}$ más básico y mayor densidad aparente, pero menor contenido de materia orgánica y salinidad del suelo. La zona 3 estuvo caracterizada por mayor contenido de materia orgánica, menor densidad aparente y $\mathrm{pH}$ del suelo, con valores de salinidad del suelo medios y por debajo del promedio.

La incorporación de otras variables hidroedáficas en el análisis no mejoró el porcentaje de discriminación correcta entre zonas y no hubo asociación entre las franjas A, B o C con alguna de las variables ambientales.

Los parámetros del sustrato que mostraron correlaciones significativas $(\mathrm{p}<0,05)$ fueron la densidad aparente con el $\mathrm{pH}$ del suelo $(\mathrm{r}=$ 0,61 , la densidad aparente con el porcentaje de materia orgánica $(\mathrm{r}=-0,59)$ y el $\mathrm{pH}$ del suelo con el porcentaje de materia orgánica $(r=-0,42)$. También hubo una alta correlación negativa entre el porcentaje de arena y de limo $(r=-0,97)$, pero esta relación se produjo porque el porcentaje de arcilla fue muy bajo (Tabla 1) y el porcentaje de arena aumentó prácticamente a expensas de la disminución del porcentaje de limo.

El ambiente y la vegetación. El Análisis de Correspondencia Canónico indicó que 26 \% de 
la variación (inercia) de este sistema es explicada por la densidad aparente, el porcentaje de materia orgánica, el pH y la salinidad del suelo $(\mathrm{p}<0,002)$.

Los dos primeros ejes canónicos (Figura 5) acumularon $96 \%$ de la variación explicada de este sistema. Los morfotipos de Sporobolus virginicus se ubicaron en extremos opuestos del diagrama (Figura 4), lo que los asocia con condiciones edáficas distintas. El morfotipo grande apareció en sustratos con pH más básico, mayor densidad aparente, menor salinidad y menor porcentaje de materia orgánica en comparación con el morfotipo pequeño. El resto de las especies mostró preferencia a alcanzar su máxima dominancia en las zonas 1 y 3 , donde hubo mayor contenido de materia orgánica y salinidad en el suelo, así como un $\mathrm{pH}$ menos básico.

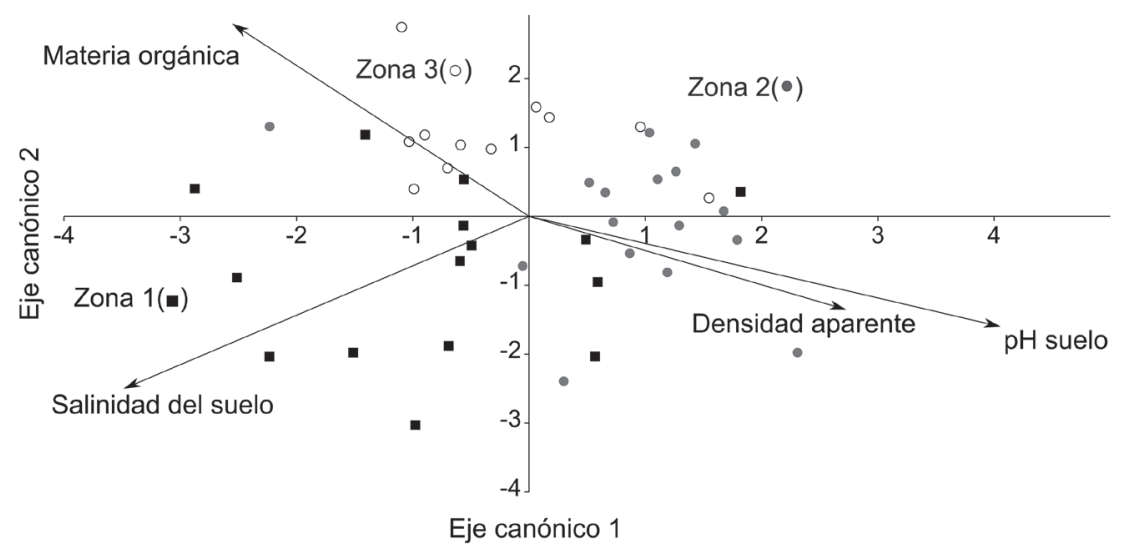

Figura 4. Biplot canónico derivado del análisis discriminante. Las unidades muestrales se representan por puntos de la zona $1(\mathbf{\square})$, zona 2 (•) y zona $3(\circ)$. Las flechas indican la dirección de aumento de las variables edáficas.

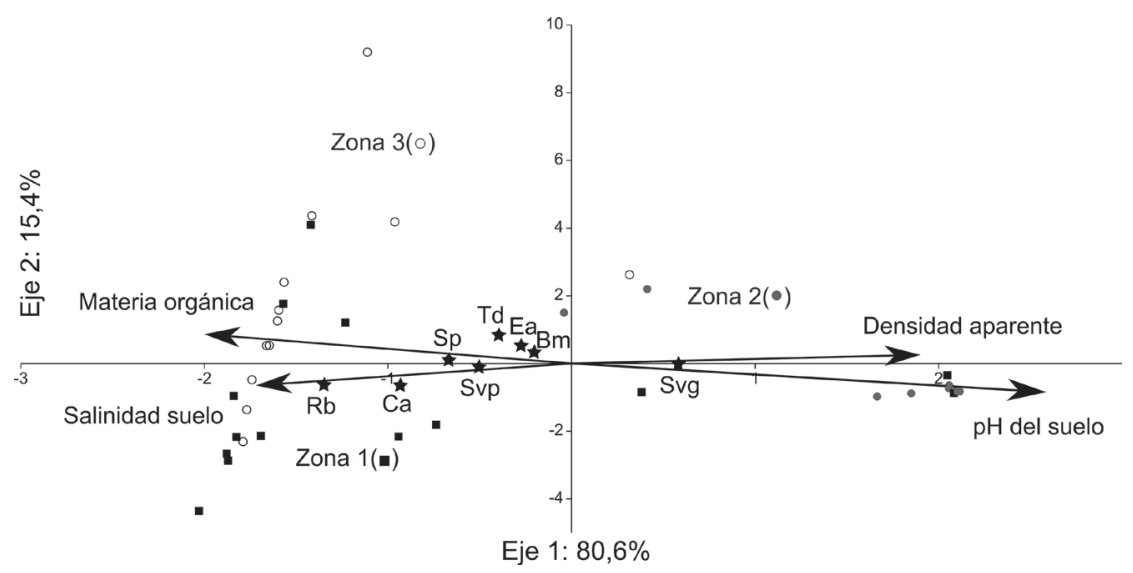

Figura 5. Diagrama de ordenación del Análisis de Correspondencia Canónica (CCA). Las unidades muestrales se representan por puntos de la zona $1(\bullet)$, zona $2(\bullet)$ y zona $3(\circ)$. Las especies $(\star)$ se abrevian: Sporobolus virginicus morfotipo pequeño (Svp), Sporobolus virginicus morfotipo grande (Svg), Eleocharis acutangula (Ea), Cyperus articulatus (Ca), Sesuvium portulacastrum (Sp), Typha domingensis (Td), Batis maritima (Bm), Rhabdadenia biflora (Rb). Las flechas indican la dirección de aumento de las variables edáficas. Los Factores de Inflación de la Varianza son: Materia orgánica: 1.37; Salinidad del suelo: 1.14; Densidad aparente: 1.33 y pH del suelo: 1.60. 


\section{Discusión}

Los resultados apoyan la hipótesis de que las condiciones del sustrato pueden determinar cambios en la comunidad vegetal y particularmente en la distribución de los dos morfotipos de Sporobolus virginicus. El transecto estudiado presentó diferencias significativas en algunos parámetros del suelo, aunque la prueba a posteriori no detectó diferencias entre sus unidades, lo que en principio resultó inconsistente. Sin embargo, el rechazo o aceptación de una hipótesis nula solo es un resultado apoyado por los datos disponibles (Daniel, 2002) que en este caso estuvieron conformados por 14 puntos de muestreo y 3 réplicas por punto, con un cierto nivel de error estándar. Al cambiar la escala de análisis, los mismos datos fueron reorganizados por zonas, considerando más réplicas por punto y reduciendo el error estándar, lo que facilita la detección de diferencias significativas, cuando existen. Más allá del argumento estadístico, la nueva escala de análisis surgió por el patrón observado en la vegetación, lo que reflejó la importancia de escoger una escala apropiada para registrar los procesos que ocurren en un ambiente natural (Kim y Zheng, 2011) y resalta el valor de la vegetación como un indicador de los cambios en el sustrato (Härdtle et al., 2006).

El transecto presentó variaciones en el suelo consistentes con otros estudios realizados en herbazales halófilos que señalan al relieve, la hidrología y la salinidad como los factores más importantes sobre los cambios en el sustrato (Breen et al., 1977; Goudkamp y Chin, 2006; Lonard et al., 2011, 2013; Guo et al., 2015). Algunos de los parámetros del suelo estuvieron correlacionados: la asociación negativa entre el $\mathrm{pH}$ y el porcentaje de materia orgánica es explicada porque a mayor contenido de materia orgánica hay mayor liberación de protones $\left(\mathrm{H}^{+}\right)$y en consecuencia menor $\mathrm{pH}$ (Ritchie y Dolling, 1985; Reddy y DeLaune, 2008), asociación que también fue encontrada en 18 humedales herbáceos del estado Miranda (Suárez-Villasmil et al., 2015). La relación negativa entre la densidad aparente y el porcentaje de materia orgánica se produce porque al aumentar los contenidos de materia orgánica en el suelo, mejora su capacidad de retener humedad y disminuye su densidad aparente (Heuscher et al., 2005; Salamanca y Sadeghian, 2005; Ruehlmann y Körschens, 2009); en consecuencia, un suelo con mayor densidad aparente presenta también $\mathrm{pH}$ más alto, como lo apoyan los resultados de este estudio.

La distribución de las especies respondió a diferencias en la densidad aparente, el pH del suelo, la salinidad y el porcentaje de materia orgánica, los cuales delimitaron tres zonas con distintas características edáficas y límites difusos entre ellas, donde se conformaron ensamblajes comunitarios distintos con algunas especies compartidas. Solo se encontró un trabajo con estudios comparables para la asociación entre los factores ambientales y composición de especies: este trabajo fue realizado en China (He et al., 2012) y sus resultados fueron muy similares a los de este estudio, determinando que los factores importantes en el herbazal halófilo conformado por ocho especies, fueron el $\mathrm{pH}$, la densidad aparente, salinidad, contenido de humedad en el suelo y el carbono orgánico. Estos resultados evidencian que puede existir similitud en la dinámica de los herbazales halófilos a pesar de encontrarse a distintas latitudes y presentar distinta composición de especies.

La asociación entre estas variables con efecto importante sobre la estructura de la comunidad hace pensar que los principales factores que originan sus cambios son la materia orgánica (con efecto sobre el $\mathrm{pH}$ y la densidad aparente del suelo) y la salinidad. La materia orgánica es producida en gran parte por las plantas emergentes (helófitas) que dominan los humedales herbáceos, de las cuales una baja proporción es consumida y asimilada por la fauna y la restante muere en pie y se incorpora a la cadena de detritus (Torres et al., 2012). La dinámica de la materia orgánica garantiza el aporte de nutrientes a estos ambientes (Neiff, 2001), porque en períodos de inundación, 
el oxígeno se agota en la lámina de agua y la descomposición es prácticamente nula. Esto produce una acumulación de nutrientes que serán liberados en forma disponible para las plantas cuando la lámina de agua se retire y el oxígeno haga posible la descomposición de la materia orgánica (Reddy y DeLaune, 2008). La salinidad, con efecto determinante en la zonación de las especies (Pennings y Callaway, 1992; Sharpe y Baldwin, 2009; Lonard et al., 2011; Guo et al., 2015) se produce como resultado de la dinámica de las lagunas La Reina y Loma del Viento, las cuales también dependen del régimen de precipitación y de evaporación de la zona. Este herbazal, dominado por Sporobolus virginicus presentó una salinidad mucho mayor a los herbazales dominados por esta planta en la isla La Tortuga, Venezuela (Marrero, 2013), donde también fue dominante en suelos con salinidades entre 2 y $14 \%$, lo que muestra su amplia tolerancia a la salinidad. En experimentos realizados con esta especie, la tasa de crecimiento así como la biomasa aérea y subterránea incrementaron con el contenido de cloruro de sodio $(\mathrm{NaCl})$ del medio de crecimiento, con salinidades máximas entre 6 y 9 \%o (Bell y O'Leary, 2003). Sin embargo, de acuerdo con el cociente molar potasio/ sodio $(\mathrm{K} / \mathrm{Na}$ ) esta especie es considerada como halotolerante (Medina et al., 2008).

La dominancia desigual de las especies refleja ausencia o muy bajo nivel de perturbaciones en esta comunidad (Connell, 1978; Dunn y Scott, 1987; Dial y Roughgarden, 1998; Svensson et al., 2012). Las especies con mayor cobertura pertenecen a las familias Poaceae y Cyperaceae, como se ha reportado en estos ambientes (Gordon, 2000; Muldavin et al., 2000; Gordon et al., 2001; GarcíaLópez et al., 2006; Suárez-Villasmil et al., 2015).

La familia Poaceae estuvo representada por Sporobolus virginicus, que fue la especie dominante, con más del $85 \%$ de la cobertura aérea (biomasa total). Esta planta se distribuye en una amplia variedad de ambientes costeros como playas, dunas arenosas, herbazales halófilos, praderas costeras o pantanos costeros (Medina et al., 2008), ya que está estimulada por la salinidad, pues elimina el exceso de sal a través de glándulas ubicadas en sus hojas, evitando la acumulación tóxica de sodio (Bell y O'Leary, 2003; Lonard et al., 2013). En este trabajo pensamos que los dos morfotipos hallados tienen similitud morfológica con las variedades minor y virginicus de S. virginicus reportadas para Estados Unidos (Lonard et al., 2013) y que Cabrera et al. (2013) reconocieron en la misma zona de estudio dos años antes del muestreo sobre el cual se basó este trabajo. Sin embargo, no podemos confirmar que los morfotipos correspondan con estas dos variedades sin el respaldo de un estudio cromosómico, lo cual escapa al alcance del proyecto de investigación.

Los dos morfotipos de Sporobolus virginicus se excluyeron y se ubicaron sobre condiciones edáficas distintas: el morfotipo pequeño (probablemente S. virginicus var. minor) estuvo asociado con suelos con menor densidad aparente, menor $\mathrm{pH}$, mayor porcentaje de materia orgánica y mayor salinidad en el suelo en comparación con el morfotipo grande (probablemente $S$. virginicus var. virginicus). La densidad aparente es la masa de un volumen de suelo seco que incluye tanto los sólidos como los poros, por ello es un indicador de la estructura, resistencia mecánica y cohesión del suelo donde aquellos con mayor densidad aparente tienen menor aireación y dificultan el crecimiento de las raíces, afectando el crecimiento de las plantas al variar la resistencia y porosidad del suelo sobre las raíces (FAO, 2009). Los valores de DA obtenidos en este trabajo $(0,9$ a $1,4 \mathrm{~g} /$ $\mathrm{cm}^{3}$ ) son considerados altos (FAO, 2009), pero las diferencias en la DA podrían ser determinantes sobre las diferencias encontradas en la distribución de los dos morfotipos. Cabrera et al. (2013) también encontraron asociación del morfotipo pequeño con suelos de menor densidad aparente, mientras que Lonard et al. (2013) relacionaron al morfotipo pequeño con dunas y al grande con pantanos.

La familia Cyperaceae apareció representada por Eleocharis acutangula y Cyperus articulatus; $E$. acutangula ha sido reportada en humedales y pantanos (Rolon y Maltchik, 2006), en asociación 
frecuente con Oxycaryum cubense (Poepp. \& Kunth) Palla (Cyperaceae) (Galán De Mera y LinaresPerea, 2008). Cyperus articulatus estuvo poco representada en el área de estudio, pero dominó el herbazal adyacente con suelos cubiertos por una lámina de agua más profunda y posiblemente con mayor permanencia, ya que esta especie se encuentra en suelos inundados por aguas dulces (Gupta et al., 2015: Cyperus articulatus, The IUCN Red List of Threatened Species 2015, http:/ / www. iucnredlist.org/) y en la colmatación de lagunas y esteros (Galán De Mera y Linares-Perea, 2008). Estas dos especies deciperáceas presentan rizomas que le facilitan la reproducción vegetativa y la formación de colonias. Las ciperáceas favorecen la formación de suelo, ya que sus culmos crecen muy cerca unos de otros y en gran número, lo que genera retención y asentamiento de la materia orgánica (Espinar et al., 2002). Además, al ser hierbas de crecimiento rápido, al igual que las poáceas, generan más cantidad de biomasa, lo que incrementa la fijación del carbono por parte del humedal.

Batis maritima L. (Bataceae) y Sesuvium portulacastrum (L.) L. (Aizoaceae) estuvieron asociadas con las parcelas A, las más cercanas al manglar. Batis maritima, aparece en amplia variedad de ambientes costeros, tolera bien la salinidad y es afectada por la inundación sostenida (Lonard et al., 2011), sin embargo, es conocida por facilitar el establecimiento de plántulas de Avicennia germinans (Milbrandt y Tinsley, 2006; Whigham et al., 2009), mientras que Sesuvium portulacastrum estabiliza los suelos y favorece la formación de dunas (Tavares-Correa y Sabogal, 2003), actuando como una barrera contra el agua y la arena (Lonard y Judd, 1997). Batis maritima y Sesuvium portulacastrum, con órganos suculentos y un cociente molar más bajo que el de Sporobolus virginicus, son consideradas como halófitas sensu stricto (Medina et al., 2008).

Typha domingensis está asociada con hábitats fértiles, perturbados y con una lámina de agua profunda $(>1,2 \mathrm{~m})$, ya que posee adaptaciones para tolerar altas salinidades, presencia de metales pesados y largos periodos de inundación (Olivares et al., 2002). Typha domingensis, además, responde favorablemente a excesos de nitrógeno y fósforo en el ambiente (Olivares et al., 2002; Vera et al, 2010; Vieira de Mendonça et al., 2012), reduciendo la eutrofización y mejorando la calidad de las aguas. Rhabdadenia biflora estuvo presente en muy baja cobertura y con solo una aparición, es una liana que tolera sustratos de baja salinidad y que está asociada con manglares, sobre los que crece como medio de soporte para facilitar su acceso a la luz (Hernández Carmona, et al. 2014).

Los resultados de este trabajo muestran cómo la tolerancia diferencial a las distintas condiciones del humedal, produce la zonación de la vegetación (Sculthorpe, 1971; Spence, 1982; Keddy, 1984; Klosowski, 1992; Pennings y Callaway, 1992; Gordon, 1998; Gordon et al., 2001; Barker y Maltby, 2009; Guo et al., 2015), debido a cambios en la composición de especies. La dinámica del agua del suelo y las condiciones microtopográficas en ambientes planos, tienen un efecto ecológicamente significativo sobre la comunidad vegetal, pues los sectores más deprimidos pueden acumular más agua que otros más elevados con efecto sobre la fertilidad y otras de las propiedades del sustrato (Martín et al., 2007). Esto hace pensar que los cambios podrían estar relacionados con mínimas variaciones en la topografía, es decir, pequeñas elevaciones y depresiones que podrían determinar diferencias en los procesos de inundación y evaporación a escala local. Marrero (2013) encontró que variaciones en la topografía explicaron diferencias observadas en las condiciones de los suelos de distintos tipos de herbazales halófilos y psamófilos. En estos herbazales, Sporobolus virginicus se ubicó sobre zonas topográficamente más elevadas, mientras que Batis maritima y Sesuvium portulacastrum fueron encontradas sobre zonas más deprimidas y cercanas al nivel freático.

\section{Conclusiones}

La tolerancia diferencial a las distintas condiciones del humedal estuvo asociada con las variaciones en la composición de especies y en particular, en 
la distribución de dos morfotipos de una misma especie. Si bien este trabajo no caracterizó el relieve detallado en cada muestra para confirmar si las diferencias en los tipos de suelo podían asociarse con variaciones en el microrelieve, los resultados muestran que el nivel de complejidad de este ambiente apunta abordar su estudio considerando no solo la medición del $\mathrm{pH}$, densidad aparente, porcentaje de materia orgánica y salinidad, sino además caracterizando detalladamente la microtopografía del relieve y su asociación con los factores edáficos, probablemente a través de la medición de la profundidad del agua superficial.

\section{Agradecimientos}

Este trabajo fue financiado por el Consejo de Desarrollo Científico y Humanístico de la Universidad Central de Venezuela (CDCHUCV), a través del Proyecto PI 7731-2009. Los autores agradecemos a Carlos Alvarado, Rossana Mendoza y a la familia Barreto por su apoyo en las actividades de campo, así como a Elizabeth Gordon, Shingo Nozawa e Ismael Hernández, por sus sugerencias al trabajo. Asimismo al Laboratorio de Ecología de Plantas Acuáticas y al Laboratorio de Ecología de la Vegetación del Instituto de Zoología y Ecología Tropical, de la Facultad de Ciencias, Universidad Central de Venezuela, por sus materiales y recursos para realizar el procesamiento de las muestras. También agradecemos a los revisores anónimos que evaluaron nuestro trabajo por sus valiosas sugerencias para la versión final.

\section{Referencias}

Afifi, A. A. y Clark, V. (1997). Computer-Aided Multivariate Analysis. Boca Ratón: Chapman y Hall. 455 pp.

Anderson, M. J. (2001). A new method for nonparametric multivariate analysis of variance. Austral Ecology, 26, 32-46.

Barker, T. y Maltby, E. (2009). The Dynamics of Wetlands (pp: 115-119). En Maltby, E. y Barker
T. (Eds.) The Wetlands Handbook. Oxford: Wiley Blackwell.

Barreto, M. B., Camaripano, B., Dorta, K., Freire, R., Manuitt, P. y Rangel, G. (2001). Composición florística y zonación de los bosques de mangle de la laguna La Reina (Higuerote, Edo. Miranda, Venezuela) (p. 264). En Programa y libro de Resúmenes del IX Congreso Latinoamericano sobre Ciencias del Mar. San Andrés, Colombia.

Bell, H. L. y O'Leary, J. W. (2003). Effects of Salinity on Growth and Cation Accumulation of Sporobolus virginicus (Poaceae). American Journal of Botany, 90, 1416-1424.

Boyoucos, G. J. (1962). Hydrometer method improved for making particles size analysis of soil. Agronomy Journal, 54, 464-465.

Breen, C. M., Everson, C. y Rogers, K. (1977). Ecological studies on Sporobolus virginicus (L.) Kunth with particular reference to salinity and inundation. Hydrobiologia, 54(2), 135-140.

Cabrera, V., Avendaño Y., Marrero M. A., Mendoza R., Pérez K., Barreto M. B. y Suárez-Villasmil L. (2013). Distribución de dos morfotipos de Sporobolus virginicus (L.) Kunth en un humedal herbáceo en aguna La Reina, Higuerote, Venezuela. En Programa y Libro de Resúmenes del X Congreso Venezolano de Ecología. Mérida,Venezuela. p. 590 .

CEOTEBM Comisión Estadal de Ordenación del Territorio del estado bolivariano de Miranda. (2010). Propuesta del plan de ordenación del territorio del estado bolivariano de Miranda. (Informe Técnico). Gobernación del estado Miranda, Los Teques. 259 pp.

Clarke, K. R. (1993).Non-parametric multivariate analysis of changes in community structure. Australian Journal of Ecology, 18, 117-143.

Connell, J. H. (1978). Diversity in tropical rain forests and coral reefs. Science, 199, 1302-1309.

Conard, H. (1905). The waterlilies: a monograph of the genus Nymphaea (428 pp). The Carnegie Institution of Washington, Baltimore. 
Daniel, W. (2002). Bioestadística: base para el análisis de las ciencias de la salud (910) pp. Limusa-Wiley, México D.F.

Dial, R. y Roughgarden, J. (1998). Theory of marine communities: the intermediate disturbance hypothesis. Ecology, 79, 1412-1424.

Dunn, C. P. y Scott, M. L. (1987). Response of wetland herbaceous communities to gradients of light and substrate following disturbance by thermal pollution. Vegetatio, 70(2), 119-124.

Espinar, J., García, L., García, P. y Toja, J. (2002). Submerged macrophyte zonation in a Mediterranean salt marsh: a facilitation effect from established helophytes? Journal of Vegetation Science, 13, 831-840.

FAO. (2009). Guía para la Descripción de los Suelos. Organización de las Naciones Unidas para la agricultura y la alimentación, Roma, 99 pp.

Figueroa, A., Contreras, M., Saavedra, B. y Espoz, C. (2016). Chilean Wetlands: Biodiversity, Endemism, and Conservation Challenges. En Finlayson, C. M., Everard M., Irvine K., McInnes R., Middleton B., van Dam A. y Davidson N. C. (Eds.). The Wetland Book. Pp. 1-17. Dordrecht: Springer Netherlands.

Galán De Mera, A. y Linares-Perea, E. (2008). Datos sobre la vegetación de los humedales de América del Sur. De las sabanas bolivianas a los llanos del Orinoco (Venezuela). Acta Botánica Malacitana, $33,271-288$.

García-López, E., Zavala-Cruz, J. y Palma-López, D. J. (2006). Caracterización de las comunidades vegetales en un área afectada por derrames de hidrocarburos. Terra Latinoamericana, 24(1), 1726.

Gordon, E. (1998). Composición fisionómica y florística de humedales dominados por Montrichardia arborescens en Laguna Grande (Monagas, Venezuela). Acta Biólogica Venezuelica, 18(1), 55-76.

Gordon, E. (2000). Dinámica de la vegetación y del banco de semillas en un humedal herbáceo lacustrino (Venezuela). Revista de Biología Tropical, 41, 25-42.

Gordon, E., Peña, C., Rodríguez, C., Rodríguez, J. y Delgado, L. (2001). Caracterización de la vegetación en un humedal herbáceo Oligohalino (Sabanas de Venturini, Sucre, Venezuela). Acta Biologica Venezuelica, 21, 41-49.

Gordon, E., Feo, Y. y Suárez-Villasmil, L. (2007). Efecto de la profundidad del agua sobre el crecimiento y biomasa de Hymenachne amplexicaulis en un humedal (estado Miranda, Venezuela). Revista de la Facultad de Agronomía, 24, 214-219.

Goudkamp, K. y Chin, A. (2006). Mangroves and Saltmarshes. Informe técnico (34 pp). The State of the Great Barrier Reef, Great Barrier Reef Marine Park Authority, Townsville.

Gratani, L. (2014). Plant phenotypic plasticity in response to environmental factors. Advances in Botany. Article ID 208747, 17 p. DOI: http:// dx.doi.org/10.1155/2014/208747.

Guo, H., Chamberlain, S. A., Elhaik, E., Jalli, I., Lynes, A. R., Marczak, L., Sabath, N., Vargas, A., Wieski, W., Zelig, E. M. y Pennings, S. C. (2015). Geographic Variation in Plant Community Structure of Salt Marshes: Species, Functional and Phylogenetic Perspectives. Plos One 2015. DOI: $10.1371 /$ journal.pone.0127781.

Gusewell, S. (2005). Responses of wetland graminoids to the relative supply of nitrogen and phosphorus. Plant Ecology, 176, 35-55.

Hammer, Ø., Harper, D. A. T. y Ryan, P. D. (2001). PAST: Paleontological Statistics Software Package for Education and Data Analysis. Palaeontología Electrónica, 4, 9.

Härdtle, W., Redecker, B. y Meyer, H. (2006). Vegetation responses to environmental conditions in flood plain grasslands: Prerequisites for preserving plant species diversity. Basic and Applied Ecology, 7(3), 280-288.

He, Q., Chen, F., Cui, B. y An, Y. (2012). Multi-scale segregations and edaphic determinants of marsh 
plant communities in a western Pacific estuary. Hydrobiología, 696, 171-183.

Hernández Carmona, S., Carmona, Díaz S. y García Orduña, F. (2014). Ecología de la liana Rhabdadenia biflora (Apocynaceae) en el manglar de Sontecomapan, Catemaco, Veracruz, México. Revista Científica Biológico Agropecuaria Tuxpan, 2(4), 836-841.

Heiri, O., Lotte, A. y Lemcke, G. (2001). Loss on ignition as a method for estimating organic and carbonate content in sediments: reproducibility and comparability of results. Journal of Paleolimnology, 25, 101-110.

Heuscher, S. A., Brandt, C. C. y Jardine, P. M. (2005). Using soil physical and chemical properties to estimate bulk density. Soil Science Society of American Journal, 69, 51-56.

Keddy, P. A. (1984). Plant Zonation on lakeshores in Nova Scotia: A test of the Resource Specialization Hypothesis. Journal of Ecology, 72(3), 797-808.

Kim, D. y Yu, K. B. (2009). A conceptual model of coastal dune ecology synthesizing spatial gradients of vegetation, soil, and geomorphology. Plant Ecology, 202, 135-148.

Kim, D. y Zheng, D. (2011). Scale-dependent predictability of DEM-based landform attributes for soil spatial variability in a coastal dune system. Geoderma, 164, 181-194.

Klosowski, S. (1992). Temporal and Spatial variation of habitat conditions in the zonation of littoral plant communities. Aquatic Botany, 43, 199-208.

Lonard, R. I y Judd, F. W. (1997). The biological flora of coastal dunes and wetlands. Sesuvium portulacastrum (L.) L. Journal of Coastal Research, 13(1), 96-104.

Lonard, R. I., Judd, F. W. y Stalter, R. (2011). The Biological Flora of Coastal Dunes and Wetlands: Batis maritima C. Linnaeus. Journal of Coastal Research, 27, (3):441-449.

Lonard, R. I., Judd, F. W. y Stalter, R. (2013). The Biological Flora of Coastal Dunes and Wetlands:
Sporobolus virginicus (C. Linnaeus) K. Kunth. Journal of Coastal Research, 29(3), 706-716.

Marrero, M. A. (2013). Condiciones edáficas de los herbazales halófitos de la isla La Tortuga Venezuela. (Trabajo de grado). Caracas: Universidad Central de Venezuela, Facultad de Ciencias, Escuela de Biología. 51 pp.

Martín, B., Sosa O., Montico, S. y Zerpa, G. (2007). Relación entre las unidades de vegetación y la microtopografía en un pastizal ubicado en un sector mal drenado de Argentina. Ciencia e Investigación Agraria, 34(2), 103-113.

Medina, E., Francisco, A. M., Wingfield, R. y Casañas, O. L. (2008). Halofitismo en Plantas de la costa Caribe de Venezuela: Halófitas y Halotolerantes. Acta Botánica Venezuelica, 31, 49-80.

Milbrandt, E. y Tinsley, M. N. (2006). The role of saltwort (Batis maritima L.) in regeneration of degraded mangrove forests. Hidrobiología, 568, 369-377.

Mitsch,W. J. y Gosselink, J. G. (2007). Wetlands. New Jersey: John Wiley y Sons Inc. 582 pp.

Muldavin, E., Durkin, P., Bradley, M., Stuevery, M. y Mehlhop, P. (2000). Handbook of Wetland Vegetation Communities of New Mexico. University of New Mexico, Albuquerque, 207 pp.

Neiff, J. J. (2001). Diversity in some tropical wetland systems of South America. En Gopal, B., Junk, W. J. y Davies, J. A. (Eds.). Biodiversity in wetlands: assessment, function and conservation. Pp. 157-186. Leiden: Backhuys Publishers.

Olivares, E., Vizcaíno, D. y Gamboa, A. (2002). Mineral nutrition of three aquatic emergent macrophytes in a managed wetland in Venezuela. Journal of plant nutrition, 25(3), 475-496.

Palmer, M. (1993). Putting things in even better order: The advantages of Canonical Correspondence Analysis. Ecology, 74(8), 2215-2230.

Pennings, S. C. y Callaway, R. M. (1992). Salt marsh plant zonation: the relative importance of competition and physical factors. Ecology, 73(2), 681-690. 
Pennings, S. C. y Bertness, M. D. (2001). Salt marsh communities. En Bertness, M. D., Gaines, S. D. y Hay, M. E. Marine community ecology. Pp. 289316. Sunderland: Sinauer Associates Inc.

Reddy, K. R. (1993). Wetland soils-opportunities and challenges. Soil Science Society of America Journal, 57, 1145-1146.

Reddy, K. R. y DeLaune, R. D. (2008) Biogeochemistry of Wetlands. Science and Applications. Boca Ratón: CRC Press.Boca Ratón. 774 pp.

Rial, A. (2009). Plantas acuáticas de los llanos inundables del Orinoco. Caracas: Editorial OrinocoAmazonas. 392 pp.

Ritchie, G. S. P. y Dolling, P. J. (1985). The role of organic matter in soil acidification. Australian Journal of Soil Research, 23(4), 569-576.

Rolon, A. S. y Maltchik, L. (2006). Environmental factors as predictors of aquatic macrophyte richness and composition in wetlands of Southern Brazil. Hydrobiología, 556(1), 221-231.

Ruelhmann, J. y Körschens, M. (2009). Calculating the Effect of Soil Organic Matter Concentration on Soil Bulk Density. Soil Science Society of American Journal, 73, 876-885.

Salamanca, J. A. y Sadeghian, K. H. (2005). La densidad aparente y su relación con otras propiedades en suelos de la zona cafetera colombiana. Cenicafé, 56(4), 381-397.

Sculthorpe, D. (1971). The Biology of Aquatic Vascular Plants. Londres, Inglaterra: Edward Arnold Ltd. Publ. 610 pp.

Sharpe, P. J. y Baldwin, A. H. (2009). Patterns of wetland plant species richness across estuarine gradients of Chesapeake Bay. Wetlands, 29(1), 225-235.

Smith-White, A. R. (1979). Polyploidy in Sporobolus virginicus (L.) Kunth. Australian Journal of Botany, $27(4), 429-437$.

Smith-White, A. R. (1988). Sporobolus virginicus (L.) Kunth in Coastal Australia: the reproductive behaviour and the distribution of morphological types and chromosome races. Australian Journal of Botany, 36(1), 23-39.
Spence,D. H. N. (1982). The zonation of plants in freshwater lakes. Advances in Ecological Research, 12, 37-125.

Suárez-Villasmil, L. M., Barreto-Pittol, E. M., Fedón, I. C., Gordon, E., García, D., Avendaño, Y. y Barreto, M. B. (2015). Riqueza, composición florística y factores hidroedáficos en humedales herbáceos de Barlovento (Estado Miranda, Venezuela). Métodos en Ecología y Sistemática, 10(2), 29-44.

Svensson, J. R., Lindegarth, M., Jonsson, P. R. y Pavia, H. (2012). Disturbance-diversity models: what do they really predict and how are they tested? Proceedings of the Royal Society, 279(1736), 2163-2170.

Tavares-Correa, C. y Sabogal, A. (2003). Estabilización de dunas litorales utilizando Sesuvium portulacastrum L. en el departamento de la libertad, costa norte del Perú. Ecología Aplicada, 2(1), 47-50.

Ter Braak, C. J. F. (1986). Canonical Correspondence Analysis: a new eigenvector technique for multivariate direct gradient analysis. Ecology, 67, 1167-1179.

Torres, R., Lugo, C., Gordon, E. y Suárez-Villasmil, L. (2012). Descomposición foliar in situ de Heliconia marginata en un humedal herbáceo (Barlovento, Venezuela). Polibotánica, 34:1-20.

van de Rijt, C. W. C. J., Hazelhoffy, L. y Blom, C. W. P. M. (1996). Vegetation zonation in a former tidal area: A vegetation-type response model based on DCA and logistic regression using GIS. Journal of Vegetation Science, 7(4), 505-518.

van Diggelen, J. M. H., Smolders, A. J. P., Visser, E. J. W., Hicks, S., Roelofs, J. G. M. y Lamers, L. P. M. (2015). Differential responses of two wetland graminoids to high ammonium at different $\mathrm{pH}$ values. Plant Biology, 18(2), 307-315.

Velázquez, J. (1994). Plantas acuáticas vasculares de Venezuela. Caracas: Colección Estudios CDCHUCV. 992 pp.

Vera, A., Andrade, C., Flores, E., Núñez, M., Cárdenas y Morales, C. E. (2010). Removal of nutrients and organic matter in a constructed 
wetland, in function of the development of the macrophyte Typha domingensis Pers. Revista Técnica de la Facultad de Ingeniería de la Universidad del Zulia, 33(2), 145-152.

Vieira de Mendonça, H., de Melo Ribeiro, C. B., Carraro Borges, A. y Rocha Bastos, R. (2012). Remoção de nitrogênio e fósforo de águas residuárias de laticínios por sistemas alagados construídos operando em bateladas. Revista Ambiente y Agua, 7(2), 75-87.

Yamilex Avendaño

Universidad Central de Venezuela, Facultad de Ciencias

Caracas, Venezuela

yamilex_@hotmail.com

\section{Irene Carolina Fedón}

Universidad Central de Venezuela, Instituto Experimental Jardín Botánico Dr. Tobías Lasser irenefedon@cyperaceaedevenezuela.org

\section{Eduardo M. Barreto-Pittol}

Universidad Central de Venezuela, Facultad de Ciencias

barreto.eduardo@gmail.com

María de los Ángeles Marrero Universidad Central de Venezuela, Facultad de Ciencias

maria.marrero.g@gmail.com

María Beatriz Barreto

Universidad Central de Venezuela, Facultad de Ciencias

maria.barreto@ciens.ucv.ve

Lourdes M. Suárez-Villasmil

Universidad Central de Venezuela, Facultad de Ciencias (autor de correspondencia) suarez.lourdes@gmail.com
Whigham, D. F., Whigham, M. C., Feller, J. C., Rodríguez, W. y King, R. S. (2009). Ecological Characteristics of Batis maritima in Florida and Belize. Smithsonian Contributions to the Marine Sciences, 38, 492-498.

Yule, G. U. (1912). On the Methods of Measuring Association between Two Attributes. Journal of the Royal Statistical Society, 75, 579.

Zar, J. (1999). Biostatistical Analysis. New Jersey: Prentice Hall. 663 pp.

Patrones espaciales en un herbazal halófilo: suelos, comunidades vegetales y plasticidad fenotípica de Sporobolus virginicus (L.) Kunth, Révis. Gramin. 1: 67 (1829) en el Estado Miranda, Venezuela

Citación del artículo: Avendaño, Y., Fedón, I. C., Barreto-Pittol, E. M., Marrero, M., Barreto, M. B. y Suárez-Villasmil, L. M. (2018). Patrones espaciales en un herbazal halófilo: suelos, comunidades vegetales y plasticidad fenotípica de Sporobolus virginicus (L.) Kunth, Révis. Gramin. 1: 67 (1829) en el Estado Miranda, Venezuela. Biota Colombiana, 19(1), 21-38. DOI: 10.21068/c2018. v19n01a02.

Recibido: 6 de junio de 2017

Aprobado: 12 de marzo de 2018 